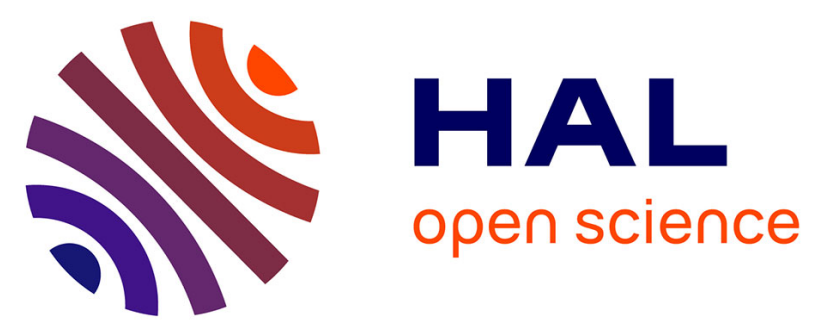

\title{
Association entre la consommation de viande rouge et de charcuteries et le risque de cancers
}

\author{
Abou Diallo, Mélanie Deschasaux, Benjamin Allès, Paule Latino-Martel, Serge \\ Hercberg, Maria del Pilar Galan Hercberg, Philippine Fassier, Françoise \\ Guéraud, Fabrice H.F. Pierre, Mathilde Touvier
}

\section{To cite this version:}

Abou Diallo, Mélanie Deschasaux, Benjamin Allès, Paule Latino-Martel, Serge Hercberg, et al.. Association entre la consommation de viande rouge et de charcuteries et le risque de cancers. Journées francophones de nutrition (JFN 2016); Montpellier (France) - (2016-11-30 - 2016-12-02) / Congrès, 2017, Montpellier, France. 10.1016/j.nupar.2017.06.024 . hal-02735108

\section{HAL Id: hal-02735108 \\ https://hal.inrae.fr/hal-02735108}

Submitted on 2 Jun 2020

HAL is a multi-disciplinary open access archive for the deposit and dissemination of scientific research documents, whether they are published or not. The documents may come from teaching and research institutions in France or abroad, or from public or private research centers.
L'archive ouverte pluridisciplinaire HAL, est destinée au dépôt et à la diffusion de documents scientifiques de niveau recherche, publiés ou non, émanant des établissements d'enseignement et de recherche français ou étrangers, des laboratoires publics ou privés. 
du cholestérol (TICE). Cependant, le cholestérol partage des voies de transport communes avec les vitamines liposolubles au niveau intestinal. Il est donc possible que le phénomène de transport inverse concerne non seulement le cholestérol mais aussi la vitamine D. Activer le TICE pourrait alors être néfaste pour les patients car la vitamine $\mathrm{D}$ est nécessaire à la prévention du risque de développer certaines pathologies osseuses, infectieuses, inflammatoires et métaboliques. Les objectifs de ce projet étaient donc d'identifier et d'évaluer la contribution respective des transporteurs membranaires apicaux responsables de la sécrétion intestinale du cholestérol et de la vitamine D, ainsi que d'évaluer si une sécrétion transintestinale peut se produire pour cette vitamine par la même voie que le TICE.

Matériel et méthodes Nous avons mis en place des approches intégrées combinant culture cellulaire (cellules HEK transfectées), études ex vivo (chambres d'Ussing) et in vivo (statut en vitamine D et expériences postprandiales) chez la souris pour évaluer l'implication de S-BI, d'ABCB1 et du complexe ABCG5/G8 dans l'efflux de vitamine D par l'entérocyte.

Résultats Les données obtenues montrent que les cellules qui surexpriment SR-BI, ABCB 1 et ABCG5/G8 ont une meilleur capacité à effluer le cholécalciférol nouvellement absorbé que les cellules témoin $(+20 \%,+32 \%$ et $+17 \%$ après $2 \mathrm{~h}$, respectivement, $p<0,05)$. In vivo, les souris déficientes en ABCB1 possèdent un statut en $25(\mathrm{OH}) \mathrm{D}$ supérieur à celui des souris sauvages, ce qui n'est pas le cas pour les souris surexprimant SR-BI au niveau intestinal. De premiers résultats obtenus avec des chambres d'Ussing confirment qu'ABCB1 serait bien impliqué dans l'excrétion transintestinale de vitamine D $(-55 \%$ de $25(\mathrm{OH}) \mathrm{D}$ effluée au travers d'explants intestinaux de souris déficientes en ABCB1 par rapport aux souris sauvages). Nos résultats postprandiaux montrent d'ailleurs que la quantité plasmatique de cholécalciférol retrouvée $3 \mathrm{~h}$ après gavage chez les souris déficientes en ABCB1 est trois fois plus importante $(p<0,05)$ que chez les souris sauvages. Une souche de souris déficientes en ABCG5/G8 au niveau intestinal est actuellement en cours de création afin de valider les résultats obtenus au niveau cellulaire.

Conclusion Nos résultats montrent que SR-BI est impliqué dans l'efflux apical de vitamine D nouvellement absorbé. ABCB1 et (probablement) ABCG5/G8 contribuent également significativement à l'efflux de vitamine D à travers l'entérocyte. Caractériser plus finement l'efflux transintestinal de la vitamine D permettra de mieux comprendre son métabolisme. Cela permettra aussi de prendre en charge de façon optimale les patients à qui seraient prescrits des activateurs de TICE, en leur proposant des recommandations nutritionnelles ou des supplémentations adaptées.

Déclaration de liens d'intérêts L'auteur déclare ne pas avoir de liens d'intérêts.

http://dx.doi.org/10.1016/j.nupar.2017.06.022

\section{CO12}

\section{Effets de l'obésité et de l'hyperglycémie du père sur le développement des cellules bêta pancréatiques et le risque de diabète dans la descendance}

K. Maouche*, L. Thomas, D. Bailbé, J. Liu, B. Portha, J. Movassat

Unité BFA, équipe biologie et pathologie du pancréas endocrine, université Paris Diderot, CNRS-UMR 8251, Paris, France

* Auteur correspondant.

Adresse e-mail : kamel.maouche@univ-paris-diderot.fr (K. Maouche)

Introduction et but de l'étude Le nombre croissant de personnes atteintes d'obésité et de diabète de type 2 a fait de ces maladies de véritables problèmes de santé publique dans notre pays et à travers le monde. Si le composant génétique et le mode de vie (alimentation non équilibrée et sédentarité) jouent un rôle majeur dans le développement de ces maladies, des modifications nutritionnelles survenues au cours de la période périnatale, sont également déterminantes dans leur pathogenèse. Cette notion est bien documentée en ce qui concerne l'impact de l'environnement maternel sur le risque de diabète et d'obésité chez la descendance à l'âge adulte, mais les données sur la programmation fœtale des maladies métaboliques induite par l'environnement paternel restent encore rares. Le but de notre projet était d'étudier l'impact de la perturbation de l'homéostasie glucidique paternelle, sur le développement fœtal et la fonction du pancréas endocrine chez la descendance.
Matériel et méthodes Nous avons tenté de répondre à cette question en utilisant deux modèles : un modèle de pré-diabète paternel induit par un régime riche en graisse, caractérisé par un surpoids associé à une intolérance au glucose, et un modèle d'hyperglycémie sévère paternelle, en absence d'obésité, induite par administration d'une drogue toxique pour les cellules $\beta$ pancréatiques. La descendance issue de chaque modèle a été étudiée à l'âge fœtal de 18,5 jours après conception, ainsi qu'à l'âge adulte de 10 semaines après la naissance.

Résultats Au stade fœtal, le diabète et le prédiabète paternels induisaient une baisse significative du poids corporel et du poids des pancréas de la descendance. L'analyse immuno-histo-morphométrique des pancréas a permis de mettre en évidence des altérations du développement du pancréas endocrine, traduites par une diminution significative de la surface relative et la masse des cellules $\alpha$ et $\beta$ pancréatiques. Cette réduction était accompagnée d'une diminution du contenu pancréatique en insuline, et d'une baisse significative de l'expression de gènes clés indispensables à la différenciation des cellules endocrines et des cellules $\beta$. Chez la descendance à l'âge adulte, le diabète paternel induit des modifications du poids corporel, et des altérations de la tolérance au glucose.

Conclusion En conclusion, notre étude a permis de mettre en évidence pour la première fois, l'effet direct de l'environnement métabolique paternel, sur les étapes précoces du développement du pancréas endocrine, et d'identifier ce défaut en tant qu'anomalie primaire, pouvant contribuer aux perturbations de l'homéostasie glucidique chez la descendance à l'âge adulte.

Déclaration de liens d'intérêts Les auteurs déclarent ne pas avoir de liens d'intérêts.

http://dx.doi.org/10.1016/j.nupar.2017.06.023

\section{Nutrition et cancer}

\section{C013}

\section{Association entre la consommation de viande rouge et de charcuteries et le risque de} cancers

A. Diallo ${ }^{1,2,3, *}$, M. Deschasaux ${ }^{2,3}$, B. Alles ${ }^{2}$, P. Latino-Martel ${ }^{2,3}$, S. Hercberg ${ }^{1,2,3}$, P. Galan ${ }^{2,3}$, P. Fassier ${ }^{2,3}$, F. Gueraud ${ }^{3,4}$, F. Pierre ${ }^{3,4}$, M. Touvier ${ }^{2,3}$

${ }^{1}$ Département de santé publique, hôpital Avicenne, Bobigny, France

${ }^{2}$ Centre de recherche en épidémiologie et biostatistique Sorbonne Paris Cité (Cress), équipe de recherche en épidémiologie nutritionnelle (Éren), Inserm U1153/Inra U1125/Cnam/Paris 13, Bobigny, France

${ }^{3}$ Réseau national alimentation cancer recherche, (Nacre), Paris

${ }^{4}$ UMR 1331 Toxalim, Inra/INP/UPS, Toulouse, France

* Auteur correspondant.

Adresse e-mail : a.diallo@eren.smbh.univ-paris13.fr (A. Diallo)

Introduction et but de l'étude Le Centre international de recherche sur le cancer (WHO-IARC) a récemment conclu à un niveau de preuve élevé concernant le caractère procarcinogène des charcuteries et viandes rouges. Ces conclusions étaient principalement basées sur le cancer colorectal, alors que la littérature scientifique est encore limitée concernant les autres localisations de cancer. L'objectif de cette étude était d'investiguer l'association prospective entre la consommation de viande rouge et de charcuteries et le risque de cancer au global, et pour les deux principales localisations en France (sein et prostate).

Matériel et méthodes Cette étude prospective incluait 61476 hommes et femmes de la cohorte NutriNet-Santé (2009-2016) âgés de 35 ans et plus et ayant complété au moins 3 enregistrements alimentaires de $24 \mathrm{~h}$ au cours des 2 premières années de suivi. Le risque de développer un cancer du sein a été comparé selon les quintiles d'apports en viande rouge et charcuteries par des modèles de Cox multivariés.

Résultats Mille six cent neuf cas de cancers incidents ont été diagnostiqués entre 2009 et 2016 , dont 544 cas de cancers du sein et 222 cas de cancer de la prostate. La consommation de viande rouge était associée à une augmentation du risque de cancers au global $\left(\mathrm{HR}_{\mathrm{Q} 5}\right.$ contre $\left.\mathrm{Q} 1=1,31(1,10-1,55), p_{\text {tendance }}=0,001\right)$ et de cancer du sein $\left(\mathrm{HR}_{\mathrm{Q} 5}\right.$ contre $\left.\mathrm{Q} 1=1,83(1,33-2,51), p_{\text {tendance }}=0,002\right)$. Cette dernière association était plus particulièrement observée chez les femmes méno- 
pausées au moment du diagnostic de cancer du sein $\left(\mathrm{HR}_{\mathrm{Q} 5}\right.$ contre $\mathrm{Q} 1=1,79$ $(1,26-2,55), p_{\text {tendance }}=0,002$, contre $p_{\text {tendance }}=0,4$ chez les femmes non ménopausées). Aucune association n'était observée entre la consommation de viandes rouges et le risque de cancer de la prostate $\left(p_{\text {tendance }}=0,9\right)$. La consommation de charcuteries, dont le niveau était relativement bas dans l'étude, n'était pas associée au risque de cancers au global et par localisation $\left(p_{\text {tendance }}>0,05\right.$ pour toutes les associations).

Conclusion Cette large étude prospective suggère que l'implication des viandes rouges dans la carcinogenèse pourrait concerner plusieurs localisations de cancer, et notamment le cancer du sein. Ces résultats sont en cohérence avec les hypothèses mécanistiques issues des études expérimentales.

Déclaration de liens d'intérêts Les auteurs déclarent ne pas avoir de liens d'intérêts.

http://dx.doi.org/10.1016/j.nupar.2017.06.024

\section{CO14}

\section{Découverte de métabolites prédictifs du}

risque de cancer du sein : approche

métabolomique RMN appliquée à

l'épidémiologie nutritionnelle

L. Lécuyer ${ }^{1,2, *}$, A. Victor Bala ${ }^{3}$, M. Deschasaux ${ }^{1,2}$, N. Bouchemal ${ }^{3}$, M. Triba ${ }^{3}$, S. Hercberg ${ }^{1,2,4}$, P. Galan ${ }^{1,2}$, E. Kesse-Guyot ${ }^{1,2}$,

P. Latino-Martel ${ }^{1,2}$, L. Fezeu ${ }^{2}$, P. Savarin ${ }^{3}$, M. Touvier ${ }^{1,2}$

${ }^{1}$ Réseau national alimentation cancer recherche (Nacre), Bobigny

${ }^{2}$ Équipe de recherche en epidémiologie nutritionnelle (Éren), centre de recherche en épidémiologie et statistiques Sorbonne Paris Cité, U1153 Inserm, U1125 Inra, Cnam, université Paris 13

${ }^{3}$ Équipe SBMB-CSPBAT-UMR 7244, université Paris 13, Bobigny cedex

${ }^{4}$ Département de santé publique, hôpital Avicenne, Bobigny, France

* Auteur correspondant.

Adresse e-mail : 1.lecuyer@eren.smbh.univ-paris13.fr (L. Lécuyer)

Introduction et but de l'étude La métabolomique est une science émergeante qui étudie l'ensemble des métabolites présents dans une cellule, un organe, un biofluide. Son application au domaine de l'épidémiologie nutritionnelle ouvre des perspectives considérables. À notre connaissance, aucune étude prospective n'avait été menée pour investiguer les liens entre les profils métabolomiques nonciblés à l'inclusion et le risque de développer un cancer du sein à long terme. Ce projet propose donc pour la lère fois d'étudier si des signatures métabolomiques établies à partir d'un simple prélèvement sanguin pourraient contribuer à mieux comprendre et à prédire le risque de cancer du sein dans la décennie suivante. Matériel et méthodes Une étude cas-témoin nichée a été mise en place dans la cohorte SU.VI.MAX (1994-2007), incluant 206 cas de cancer du sein et 396 témoins appariés. Les profils métabolomiques RMN ont été établis sur des échantillons de plasma prélevés à l'inclusion (donc avant l'apparition des cancers). Des régressions logistiques conditionnelles multivariées ont été utilisées. La performance prédictive des modèles a été évaluée grâce au NRI (Net Reclassification Improvement).

Résultats Deux-cent-trente-sept buckets ont été obtenus après division du spectre RMN par « intelligent bucketing », dont 25 étaient significatifs dans les modèles logistiques pour la séquence NOESY (respectivement 228 buckets et 27 significatifs pour la séquence CPMG). Les probabilités critiques correspondantes allaient de 0,00007 (pour le bucket 5,1869 ppm, correspondant au groupement méthine du glycéryl, $\mathrm{OR}_{\mathrm{T} 3}$ contre $\left.\mathrm{T} 1=0,37[0,23-0,61]\right)$ à 0,04 (pour le bucket 2,429 ppm, correspondant à la glutamine, $\mathrm{OR}_{\mathrm{T} 3 \text { contre } \mathrm{T} 1=1,62}$ $[1,02-2,57]$ ). Des lipoprotéines, des lipides (dont des acides gras insaturés et des glycérides et/ou des phosphoglycérides et dérivés) et des glycoprotéines étaient associées à une diminution du risque de développer un cancer du sein alors que plusieurs acides aminés et dérivés (valine, leucine, glutamine, créatine, créatinine et la thréonine) et le bêta-glucose étaient associés à une augmentation du risque. La plupart de ces métabolites augmentaient significativement la performance prédictive des modèles.

Conclusion Cette étude pionnière suggère que plusieurs métabolites, dont certains appartenant au food metabolome, seraient impliqués dans l'étiologie du cancer du sein. Des analyses similaires en métabolomique par spectrométrie de masse sont en cours dans l'étude, ainsi que l'étude des corrélations entre les profils métabolomiques et les apports nutritionnels.
Déclaration de liens d'intérêts Les auteurs déclarent ne pas avoir de liens d'intérêts.

Remerciements Institut national du cancer (INCa)/Institut fédératif de recherche biomédicale (IFRB).

http://dx.doi.org/10.1016/j.nupar.2017.06.025

\section{CO15}

Apports alimentaires, via les compléments

alimentaires et totaux en antioxydants et risque de cancers digestifs dans la cohorte prospective NutriNet-Santé

M. Touvier ${ }^{1,2, *}$, M. Egnell ${ }^{1}$, P. Fassier ${ }^{1,2}$, L. Lécuyer ${ }^{1,2}$, S. Hercberg ${ }^{1,2,3}$, P. Latino-Martel ${ }^{1,2}$, M. Deschasaux ${ }^{1,2}$

${ }^{1}$ Équipe de recherche en épidémiologie nutritionnelle (Éren), Centre de recherche en épidémiologie et statistiques Sorbonne Paris Cité, U1153 Inserm, U1125 Inra, Cnam, Université Paris 13

${ }^{2}$ Réseau national alimentation cancer recherche (Nacre)

${ }^{3}$ Département de santé publique, hôpital Avicenne, Bobigny, France

* Auteur correspondant.

Adresse e-mail : m.touvier@eren.smbh.univ-paris13.fr (M. Touvier)

Introduction et but de l'étude De nombreuses études expérimentales ont montré un effet bénéfique des antioxydants dans la prévention des cancers digestifs. Toutefois, les études épidémiologiques restent contrastées. En outre, relativement peu disposent des doses d'apport incluant une mesure quantitative de la consommation via les compléments alimentaires. L'objectif était d'étudier les associations entre les apports en vitamines $\mathrm{C}$ et $\mathrm{E}$, bêta-carotène et sélénium (alimentaires, via les compléments alimentaires et totaux) et le risque de cancers digestifs, ainsi qu'une potentielle modulation par l'apport en alcool et le statut tabagique.

Matériel et méthodes Cette étude prospective incluait 38812 sujets âgés de 45 ans et plus, de la cohorte NutriNet-Santé. Cent-soixante-sept cas de cancers digestifs incidents (120 côlon-rectum, 26 pancréas, neuf oesophages, sept estomacs et cinq foies) ont été diagnostiqués entre 2009 et 2016. Les données alimentaires étaient recueillies par des enregistrements de 24 h répétés. Un questionnaire spécifique estimait la consommation de compléments alimentaires sur une période de 12 mois. Une base de données de composition contenant 8000 compléments a été développée. Les associations entre apports en antioxydants et risque de cancers digestifs ont été caractérisées par des modèles de Cox multivariés.

Résultats et Analyse statistique Les apports en vitamine $\mathrm{C}$ ali-

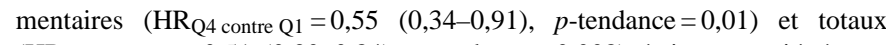
$\left(\mathrm{HR}_{\mathrm{Q} 4}\right.$ contre $\mathrm{Q} 1=0,51(0,30-0,84), p$-tendance $\left.=0,008\right)$ étaient associés à une diminution du risque de cancers digestifs, de même pour les apports en vitamine $\mathrm{E}$ alimentaires $\left(\mathrm{HR}_{\mathrm{Q} 4}\right.$ contre $\mathrm{Q} 1=0,56(0,34-0,92), p$-tendance $\left.=0,005\right)$ et totaux $\left(\mathrm{HR}_{\mathrm{Q} 4 \text { contre.Q1 }}=0,58(0,36-0,94), p_{\text {tendance }}=0,003\right)$ et les apports en sélénium alimentaires $\left(\mathrm{HR}_{\text {per } 1 \text {-unit increment }}=0,99(0,98-1,00), p=0,04\right)$ et totaux $\left(\mathrm{HR}_{\text {per I-unit increment }}=0,99(0,98-1,00), p=0,03\right)$. Des interactions significatives entre les apports alimentaires et totaux en sélénium et l'apport en alcool, et entre l'apport total en vitamine $\mathrm{E}$ et le statut tabagique ont été observées.

Conclusion Cette large étude prospective, incluant une évaluation quantitative des apports via les compléments alimentaires, suggère un possible effet protecteur des antioxydants (vitamines $\mathrm{C}$ et $\mathrm{E}$ et sélénium) sur le risque de cancers digestifs. Elle suggère également une modulation de certaines de ces relations par la consommation d'alcool et le statut tabagique.

Déclaration de liens d'intérêts Les auteurs déclarent ne pas avoir de liens d'intérêts.

Remerciements Cancéropôle Île-de-France/Région Île-de-France.

http://dx.doi.org/10.1016/j.nupar.2017.06.026

\section{CO16}

Hypermétabolisme chez le patient âgé atteint de cancer-une étude pilote

G. Ulmann ${ }^{1,2, *}$, A. Jouinot ${ }^{3}$, C. Tlemsani ${ }^{3}$, N. Neveux ${ }^{1,2}$, J.-P. Durand ${ }^{3}$,

F. Goldwasser ${ }^{3}$, J.-P. De Bandt ${ }^{1,2}$, L. Cynober ${ }^{1,2}$

${ }^{1}$ Service de biochimie, hôpital Cochin, HUPC, AP-HP 